\title{
Influence of high hydrostatic pressure on the optical properties of minced fish proteins
}

\author{
Valeriy Drobotko ${ }^{1}$, Gennadiy Bukin ${ }^{1}$, Sergey Sokolov, ${ }^{2,}$, Alexander Yashonkov ${ }^{2}$, and \\ Alexander Falko ${ }^{2}$ \\ ${ }^{1}$ Donetsk Institute for Physics and Engineering named after A.A. Galkin, str. R. Luxemburg 72, \\ Donetsk, 83114, Ukraine \\ ${ }^{2}$ FSBEI HE "Kerch State Maritime Technological University", str. Ordzhonikidze, 82, Republic of \\ Crimea, Kerch, 298309, Russia
}

\begin{abstract}
In the functioning of various biological systems, an extremely important role is played by the dynamic properties of optically anisotropic protein macromolecules, such as translational movements, temporal correlations, and transfer processes, which are determined by the surface charge, molecular weight, molecular shape, and the nature of intermolecular interactions.
\end{abstract}

\section{Introduction}

An experimental study of the influence of high hydrostatic pressure on the optical properties of pike perch minced fish proteins at room temperature was carried out in this work. The intensities of the radiation transmitted through a thin plane-parallel layer of minced fish were measured, which was subjected to hydrostatic compression at a rate of $\approx 3$ $\mathrm{MPa} / \mathrm{s}$ and $\approx 17 \mathrm{MPa} / \mathrm{s}$. It was found that with increasing pressure, the intensity of the transmitted light decreases nonmonotonically; in this case, the experimental plots of the $I(P)$ dependence show the presence of broad attenuation bands of the intensity of the radiation transmitted through the sample, located at pressures of about 150,300 , and 700 $\mathrm{MPa}$. The observed attenuation bands are associated with the appearance of scattering centers formed as a result of denaturation of macromolecules of proteins of various types with different functional purposes contained in the muscle tissue of pike perch. It was found that the duration of the action of hydrostatic pressure affects the processes of denaturation of protein macromolecules, which is caused by the dependence of the transfer of water into the protein in the bulk backbone of the particles of muscle fibers of minced fish on the duration of hydrostatic compression. As a result, in a time equal to $\approx 60 \mathrm{~s}$, when the sample is compressed at a rate of $\approx 17 \mathrm{MPa} / \mathrm{s}$, there are $\approx 20 \%$ less denatured protein macromolecules than in a time equal to $\approx 335 \mathrm{~s}$, when the sample is compressed at a rate of $\approx 3 \mathrm{MPa} / \mathrm{s}$.

In the functioning of various biological systems, an extremely important role is played by the dynamic properties of optically anisotropic protein macromolecules, such as

\footnotetext{
*Corresponding author: sergiiblack@gmail.com
} 
translational movements, temporal correlations, and transfer processes, which are determined by the surface charge, molecular weight, molecular shape, and the nature of intermolecular interactions.

Aqueous solutions of protein macromolecules are unique objects for studying these characteristics using optical methods, for example, using Rayleigh light scattering [1].

As a result of the interaction of the surface groups of a protein molecule with water, the tensor of electronic polarizability changes and, accordingly, polarization studies of the protein molecule are also of interest. It was shown in [2] that the polarization characteristics of albumin, gamma-globulin and fibrinogen molecules in aqueous solutions significantly depend on the surface charge of the protein macromolecule. The surface charge of a molecule is determined by the concentration of free hydrogen ions in the solution and can reach zero [3].

Pathological processes in living organisms are accompanied by changes in a number of molecular parameters in cells, tissues and blood serum. Therefore, studies of the behavior of protein macromolecules in solutions are important for understanding these processes.

To study them, effective optical methods of static and dynamic light scattering and methods of direct determination of the sizes and shapes of macromolecules and their aggregates are used [4].

The influence of external factors on proteins, as the main constituent elements of living nature, is one of the most pressing problems in such fields of science as biophysics, microbiology, medicine, ecology and food industry.

Under the action of external hydrostatic pressure, such parameters of protein molecules as volume, surface area, total surface charge, optical anisotropy, polarizability tensor change, and the coefficients of intermolecular interaction, translational and rotational diffusion can also change significantly [5].

The aim of this work is an experimental study of the influence of high hydrostatic pressure on the optical properties of minced fish proteins at room temperature by means of light attenuation.

\section{Materials and methods of research}

\subsection{Materials}

The object of the study was minced fish from pike perch meat. Minced fish is an isotropic suspension of understained and weakly scattering polydisperse unbound particles of destroyed muscle tissue (dispersed phase) in an intercellular transparent liquid (dispersion medium). The understaining of minced fish in the visible spectral range is caused by the significant absorption of proteins in the ultraviolet region in the wavelength range from 180 to $310 \mathrm{~nm}[6]$.

The main proteins of fish muscle tissue are myofibrillar and sarcoplasmic proteins. Myofibrillar proteins make up from 65 to $80 \%$ of the total protein content and consist mainly of contractile proteins (actin and myosin), as well as regulatory, elastic, and some other minor proteins.

To obtain mince, the fish was scaled and cut. Then, the muscle tissue was separated from the skin and bones, passed through an electric meat grinder with a grid hole diameter of $2 \mathrm{~mm}$, and thoroughly milled in a blender.

After denaturation of proteins by hydrostatic pressure [7], the minced fish becomes white and opaque, which makes it possible to use the method of light attenuation in the experiment. For this purpose, using a round metal ring, the samples were formed in the 
form of a thin plane-parallel layer with a thickness of $l=0.2 \mathrm{~mm}$, held together by two plates of adhesive tape.

\subsection{Method of exposure to ultra-high pressure on the sample}

A sample of minced fish prepared for measurements was placed in a cuvette of a highpressure liquid optical chamber (HPOC) so that the radiation flux fell on the sample normally (perpendicular to the plane of the minced fish layer). Using an automated highpressure setup (AHPS) [8], the working fluid was hydrostatically compressed with the sample in the HPOC cuvette.

The pressure of the working fluid acting on the sample was increased in the range from atmospheric to $1000 \mathrm{MPa}$ at a room temperature of $300 \mathrm{~K}$. In one experiment, the rate of pressure increase was about $17 \mathrm{MPa} / \mathrm{s}$, and in the second, $3 \mathrm{MPa} / \mathrm{s}$. Upon reaching 1000 $\mathrm{MPa}$, the pressure was automatically reduced to atmospheric pressure within $30 \mathrm{~s}$.

The intensities of the radiation incident and transmitted through the sample at a wavelength $\lambda=555 \mathrm{~nm}$ were recorded on an upgraded PGS-2 spectrograph with PMT-118. The transmitted radiation intensity was normalized to the incident radiation intensity. The current time of the experiment, the pressure acting on the sample, and the intensity of the radiation that passed through the sample were automatically documented in real time.

\subsection{Methods of light absorption and attenuation}

The absorption of electromagnetic radiation passing through an absorbing transparent solution depends on the concentration and electronic composition of atoms and molecules of the solute, the wavelength of the incident radiation, the thickness of the layer, and external physical parameters such as temperature and pressure.

To quantitatively describe the process of absorption of transmitted radiation, the Bouguer - Lambert - Beer law is used, which relates the thickness of the solution layer $l$ (optical path length) through which the radiation flux passes, the concentration of absorbing atoms and molecules in the solution $c$ with the intensities of the incident $I_{0}$ and transmitted $I$ radiation.

For monochromatic radiation, the Bouguer - Lambert - Beer law in differential form has the form [9]:

$$
-d I=k \cdot c \cdot I_{0} \cdot d x
$$

where $k$ - absorption coefficient, which characterizes the ability of a solution to reduce the intensity of transmitted radiation as a result of absorption of dissolved atoms and molecules, $c$ - concentration of absorbing atoms and molecules in solution, $-d I-$ the amount by which the radiation intensity decreases (minus sign) when it passes through the solution a path with a length $d x, I_{0}-$ incident radiation intensity.

Integration of equation (1) in the range from $I_{0}$ to $I$ and from $x=0$ to $x=l$ gives the expression for the optical density $D$ :

$$
D=\ln \left(\frac{I_{0}}{I}\right)=k \cdot c \cdot l
$$

which is a measure of the decrease in radiation intensity when it is absorbed by a solution. 
In the method of attenuation of light passing, for example, through a colloidal solution, the decrease in the radiation intensity depends on the same parameters as in the method of light absorption. However, in this case, the decrease in the radiation intensity at the scattering centers in the solution is also taken into account.

In this case, the Bouguer - Lambert - Beer law (1) in differential form takes the form:

$$
-d I=\varepsilon \cdot c \cdot I_{0} \cdot d x
$$

where $\varepsilon=k+r-$ attenuation (extinction) coefficient, which characterizes the ability of a scattering and absorbing solution to reduce the intensity of transmitted radiation as a result of absorption (absorption coefficient $\boldsymbol{k}$ ) and scattering (scattering coefficient $r), c=c_{k}+c_{r}$ - the sum of the concentrations of absorbing atoms and molecules in the solution $c_{k}$ and the concentration of scattering centers $c_{r}$.

\section{Results and discussion}

Equation (3) is the basis of the method of attenuation of the light flux when it passes through the scattering and absorbing system. The characteristics of minced fish considered above allow assuming in the first approximation that in the visible spectral range, the sample under study almost does not absorb light, i.e. the absorption coefficient is taken to be zero $k \approx 0$, and we assume that the weakening of the light flux occurs only due to the processes of light scattering, i.e. attenuation coefficient $\varepsilon \cong r$.

For the correct application of this method, two necessary conditions must be met: the absence of absorption and secondary light scattering in the sample. The first condition is satisfied by choosing a wavelength in the visible spectral region, where the sample does not absorb light. To reduce the effect of multiple scattering of light, a plane-parallel layer thickness equal to $l=0.2 \mathrm{~mm}$ was used.

The dependences of the measured intensity $I$ of radiation transmitted through the sample during its compression at a rate of $\approx 17 \mathrm{MPa} / \mathrm{s}(1)$ and $\approx 3 \mathrm{MPa} / \mathrm{s}(2)$ at a temperature of 300 $\mathrm{K}$ and the calculated natural optical density $D$ on the hydrostatic pressure $\mathrm{P}$ acting on the sample are shown in figure 1.

Figure 1 shows that with increasing pressure, the intensity of the transmitted light decreases nonmonotonically. At pressures of about 150,300, and $700 \mathrm{MPa}$, the curves have clearly visible broad attenuation bands of the transmitted light intensity, which means the appearance of scattering centers in the sample reflecting the process of denaturation of protein macromolecules in minced fish under the action of applied hydrostatic pressure. With a high degree of probability, it can be assumed that the observed bands of attenuation in intensity are associated with the processes of denaturation of various types of proteins with different functional purposes contained in the muscle tissue of pike perch. 


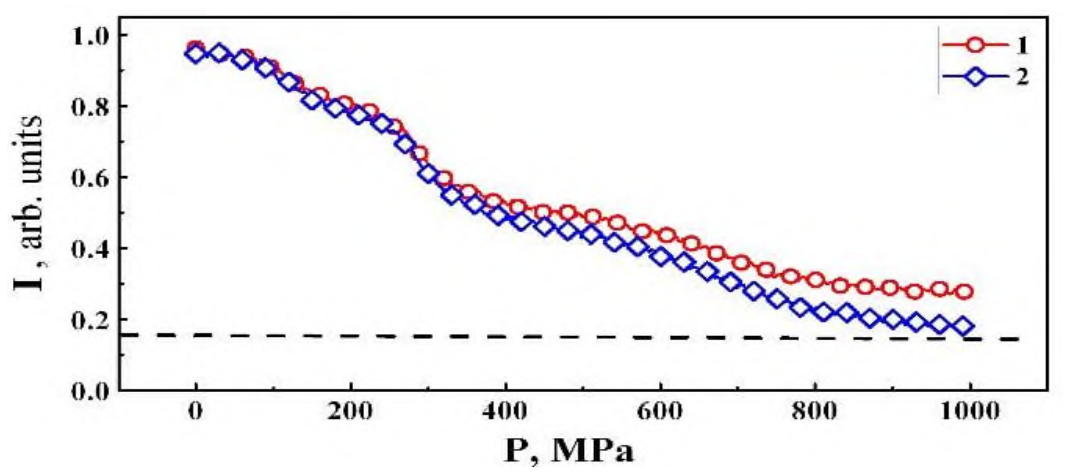

a)

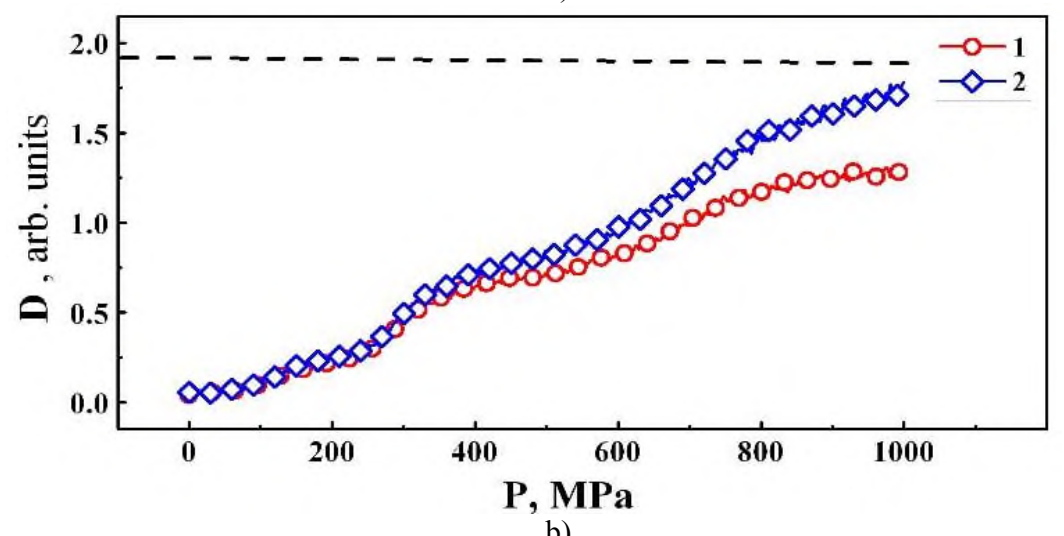

b)

Fig. 1. Dependences of the measured intensity of radiation transmitted through the sample during its compression at a rate of $\approx 17 \mathrm{MPa} / \mathrm{s}(1)$ and $\approx 3 \mathrm{MPa} / \mathrm{s}(2)$ at a temperature of $300 \mathrm{~K}$ (a) and the calculated natural optical density (b) on the hydrostatic pressure acting on the sample. The dashed line corresponds to heat-treated minced fish.

The $I(P)$ dependences measured over different times in two experiments, depending on the compression rate, show the effect of the duration of the action of hydrostatic pressure on the amount of denatured protein macromolecules. The suggested mechanism of high pressure-induced protein denaturation is the pressure-dependent transport of water into the protein. The inner space of the protein is mostly filled with packed hydrophobic residues. An increase in hydrostatic pressure forces water molecules to penetrate into the protein, gradually filling the "cavities", which ultimately leads to the destruction of the structure of the protein molecule [10]. Denaturation under pressure corresponds to the incorporation of water into the protein, while thermal denaturation corresponds to the transfer of non-polar groups to water. Obviously, the gradual filling of the protein "cavities" in the bulk backbone of the minced fish muscle fiber particles with water molecules will lead to the dependence of the denatured protein amount on the time of exposure to hydrostatic pressure [11-15]. Thus, during the experiment time equal to $\approx 60 \mathrm{~s}$, when the sample is compressed at a rate of $\approx 17 \mathrm{MPa} / \mathrm{s}$, there are approximately $20 \%$ less denatured protein macromolecules than in a longer experiment equal to $\approx 335 \mathrm{~s}$, when the sample is compressed at a rate of $\approx 3$ $\mathrm{MPa} / \mathrm{s}$. 


\section{Conclusion}

1. An experimental study of the influence of high hydrostatic pressure on the optical properties of minced fish proteins from pike perch meat at room temperature of $300 \mathrm{~K}$ was carried out by the method of light attenuation.

2 . The intensities of radiation passed through a thin plane-parallel layer of minced fish were measured, which was subjected to hydrostatic compression at a rate of $\approx 3 \mathrm{MPa} / \mathrm{s}$ and $\approx 17 \mathrm{MPa} / \mathrm{s}$.

3. It was found that with increasing pressure, the intensity of the transmitted light decreases nonmonotonically; in this case, the experimental plots of the $I(P)$ dependence show the presence of broad attenuation bands of the intensity of the radiation transmitted through the sample, located at pressures of about 150, 300, and $700 \mathrm{MPa}$. The observed attenuation bands are associated with the appearance of scattering centers formed as a result of denaturation of macromolecules of proteins of various types with different functional purposes contained in the muscle tissue of pike perch.

4. It was found that the duration of the action of hydrostatic pressure affects the processes of denaturation of protein macromolecules, which is caused by the dependence of the transfer of water into the protein in the bulk backbone of the minced fish muscle fiber particles on the duration of hydrostatic compression. As a result, in a time equal to $\approx 60 \mathrm{~s}$, when the sample is compressed at a rate of $\approx 17 \mathrm{MPa} / \mathrm{s}$, there are $20 \%$ less denatured protein macromolecules than at a time equal to $\approx 335 \mathrm{~s}$, when the sample is compressed at a rate of $\approx 3 \mathrm{MPa} / \mathrm{s}$.

\section{References}

1. K.S. Shifrin, Light scattering in a turbid environment, GI TTL (State publishing house of technical and theoretical literature, Moscow-Leningrad, 1951)

2. P. Debye, Journal Appl. Phys., 15, 338-349 (1944)

3. M. Kosmulsky, Surface charging and zero charge points (CRC Press; 1st edition (hardcover), 2009) ISBN 978-1-4200-5188-9

4. I.P. Ashmarin, A.A. Mulberg, N.V. Sadikova, S.E. Tukachinsky, Protein chemistry, in 2 parts (1968)

5. A.Kh. Vorobiev, Diffusion problems in chemical kinetics (Moscow State University, 2003)

6. D. Lakovich, Fundamentals offluorescence spectroscopy (Mir, Moscow, 1986)

7. W. Kauzmann, Nature, 325, 763-764 (1987)

8. G.V. Bukin, G.G. Levchenko, A.I. Kasyanov, et al., Inter. conf. "Functional Materials ICFM - 2007”: abstr. book (Partenit, Ukraine, 282, 2007)

9. M.M. Gurevich, Photometry. Theory, methods and devices (Energoatomizdat, 1983)

10. G. Hummer, S. Garde, A.E. Garcia, ME. Paulaitis, L.R. Pratt, Proceedings of the National Academy of Sciences, 95(4), 1552-1555 (1998)

11. S. Chernyi, V. Budnik, Methods for optimizing solutions when considering group arguments by team of experts (2017) DOI: 10.1063/1.5009873

12. S. Sokolov, A. Zhilenkov, S. Chernyi, A. Nyrkov, D. Mamunts, Symmetry, 11(2), 236 (2019) DOI: 10.3390/sym11020236 
13. J. Martin, T. Reess, A. De Ferron, R. Ruscassié, and F. R. Bethbeder, 2012 IEEE International Power Modulator and High Voltage Conference (IPMHVC), San Diego, CA, 370-373 (2012) DOI: 10.1109/IPMHVC.2012.6518757

14. T. R. Wolinski, and W. J. Bock, IEEE Transactions on Instrumentation and Measurement, 44(3), 08-711 (1995) DOI: 10.1109/19.387314

15. X. Liu, X. Peng and X. Meng, 2012 Asia-Pacific Power and Energy Engineering Conference, Shanghai, 1-6 (2012) DOI: 10.1109/APPEEC.2012.6306984 